\title{
A New Apparatus for the Measurement of Force-Frequency Effect of Crystal Resonators
}

\author{
Thanh Tuong Pham andHaifeng Zhang \\ Department of Engineering Technology, University of North Texas, Denton, TX 76207, USA
}

\begin{abstract}
We describe an apparatus for the measurement of the force-frequency effect of crystal resonators. The force-frequency effect is the correlation between the force put on the crystal resonator and the relative resonantfrequency of the resonator to the input force. The new apparatus has been designed and manufactured to measure the force sensitivity coefficient $\mathrm{K}_{\mathrm{f}}(\mathrm{m} . \mathrm{s} / \mathrm{N})$, which relates to parameters such as crystal geometry, orientation angle, and force direction (azimuth angle).The new loading device has a better force resolution and is simpler to adjustfor force alignment than traditional level-loading devices. In order to validate this new loading device, sample experiment tests have been performed, and a comparison of the experiment outcomeswith peers' measurement results show a good agreement.
\end{abstract}




\section{INTRODUCTION}

Crystal oscillators are essential components in modern electronics systems for surveillance, communication, navigation, airborne, and vehicular applications. The performance of such systems depends on the stability and accuracy of crystal oscillators. However, the operational frequency of the crystal resonator, the core of the crystal oscillator, is sensitive to external forces caused by acceleration. This unwanted sensitivity,named acceleration sensitivity,leads to excessive phase noise, which in turn degradesan electronic system'sperformance. For instance, modern GMTI (Ground Moving Target Indicator) radar uses a moving target's Doppler radar return to distinguish it from surface clutter, while the Minimum Detectable Velocity (MDV) is primarily determined by the phase noise level of a crystal oscillator. Excessive oscillator phase noise will downgrade the GMTI's ability to detect moving objects. The acceleration sensitivity of a crystal resonator is closely related to the forcefrequency effect, which refers to the phenomenon of frequency changes due to the stress applied to a resonator [1], [2]. The measurement of this effect is critical for the design of mounting supports for resonators so that the acceleration sensitivity canbe minimized. Furthermore, this measurement could provide insights to solvingthe long-term aging problem associated with resonators [3].From another perspective, the measurement of this effect could provide an important reference for force sensor design[4], [5].Therefore, the force-frequency effect on crystal resonator is the critical factor in frequency control and force measurement. Based on the fore-frequency coefficient $\mathrm{K}_{\mathrm{f}}$ of a crystal resonator, we can control the frequency more efficiently and can design a force sensor or pressure sensor with expected accuracy and precision.Experimental research on the force-frequency effect for quartz resonators has been performed for many years. Ratajski [6] measured the force-frequency effect of several singly 
rotated quartz crystal resonators. Ballato and EerNisse [3], [7] measured the force-frequency effect for doublyrotated quartz resonators. The first measurement of the force-frequency effect in langasite was published by Boy, et al. in 2001 [8], and the force-frequency effect of langanite (LGN) resonators was subsequently measured by Kim and Ballato [9]. The loading device is important for accurate measurement of the force-frequency effect, however, the aforementioned experiments use a loading device that relies on weight step loads, which is not reliable and is difficult adjust for force alignment, which may lead to inaccurate results.As will be further describedin this article, we designed and fabricated an apparatus for the measurement of the force-frequency effect of crystal resonators. The new loading device has a better input force increment $(0.01 \mathrm{~N})$ and is simpler to adjustfor force alignment than traditional level loading devices. In order to validate this new loading device, sample experiment tests have been performed, and a comparison of the experiment outcomes with peers' measurement results show a good agreement.

The paper is organized as follows: the next Sectiondescribes the basic principle underlying the experiments, and Section III offers details about the experimental assembly. The measurement procedure is illustrated in Section IV, and typical experimental results are shown in Section V. Finally, Section VI concludesthis paper.

\section{BASIC EXPERIMENTAL PRINCIPLE}

The prime parameters affecting the forcesensitivity of circular quartz crystal are identified as the orientation angle, direction of force, crystal geometry, operating frequency, and the design of the crystal holder [10].

As illustrated in Fig. 1, azimuth angle $\psi$ is the angle between the applied force direction and the crystallographic axis of a singly rotated cut. A more thorough theoretical analysis of the 
force-frequency effect for doubly rotated langasite resonators can be found in [11]. The forcefrequency effect produced in a circular crystal plate acted upon by diametric in-plane force at the angle $\psi$ is characterized by means of a force-frequency coefficient $K_{f}(m . s / N)$, which is defined by:

$$
K_{f}=\frac{\Delta f}{f} \cdot \frac{(\text { Diameter })(\text { Thickness })}{(\text { Force })(\text { Aucoustic velocity } / 2)}
$$

where $\frac{\Delta f}{f}$ is the normalized frequency change due to the applied force [9].

It has been determined that the sensitivity of the resonator frequency can be reduced by selecting a certain support orientation. The value of $\mathrm{K}_{\mathrm{f}}(\mathrm{m} . \mathrm{s} / \mathrm{N})$ can be positive, negative,or zero.

When the force-frequency relation is identified at a certain azimuth angle, this relation is linearized, and the slope of the line can be determined. The coefficient $\mathrm{K}_{\mathrm{f}}$ can also be calculated by thefollowing formula:

$$
K_{f}=\frac{d f}{d F} \cdot \frac{D}{f_{0}^{2}}
$$

wheredf $/ \mathrm{dF}$ is the slope of the line (Hz/Newton), D is the diameter of the resonator (m), and $\mathrm{f}_{0}$ is the frequency $(\mathrm{Hz})$ without force (0 Newton) [6].The sign convention for $\mathrm{K}_{\mathrm{f}}$ is defined as follows: if $\mathrm{K}_{\mathrm{f}}>0$, the frequency is increased when the applied force on the crystal resonator is increased; if $\mathrm{K}_{\mathrm{f}}<0$, the frequency is decreased when the applied force on the crystal resonator is increased; if $\mathrm{K}_{\mathrm{f}}=0$, the frequency does not change when the applied force on the crystal resonator is increased or decreased.

\section{EXPERIMENTAL APPARATUS}

Theschematic of the new loading device assembly is shown in Fig. 2. In this device, the circular crystal resonator is placed between two wedge-shape clamps(108) and (109), and the 
force is horizontallyapplied to the crystal by (109in Fig. 2)when rotating a screw (203in Fig. 2)which pushes the (109) along sliding guide rails (105in Fig. 2). Aluminum plates (101, 102, 103, and 104 in Fig. 2)that function as supports are assembled by bolt connections. Fig. 3 illustrates an isometric view of a 3D model of the apparatus.

The magnitude of the force is increased or decreased by rotating the screw. The applied force is measured by a load cell model WMC $110 \mathrm{~N}$-Interface, and the force magnitude is displayed in a Digital indicator model 9820-Interface.

Handling and mounting the crystal resonator, whichis a delicate and precise component, must be performed carefully and accurately in order to avoid damaging the resonator and to obtain precise and consistent measurement data. The direction of the force applied on the resonator must be diametric in-plane along a specific direction defined by the azimuth angle. In addition, we must ensurethat the plane of the resonator is vertical and parallel with the force direction. The advantages the new loading device has over the traditional loading device are high force resolution $(0.01 \mathrm{~N})$, accuracy and ease for force alignment, and more reliable and accurate measurement results.

\section{EXPERIMENTAL MEASUREMENT PROCEDURE}

Fig. 4 illustrates a connection setup of the experiment. Firstly, we define and mark the azimuth angles on the quartz crystal resonator. Then we connect two electrodes of the crystal resonator to the input and the output port of the network analyzerAgilent E5061B by the resonator holder. The signal from the network analyzer will be processed and displayed on the monitor of the network analyzer.Fig. 5 shows a typical frequency spectrumof a crystal resonator at 2.33 MHz.We mount the crystal on the apparatus and weensure that the crystal plane is vertical and the two clamps are diametric in-plane at the azimuth angle. The resonance frequency 
at $0 \mathrm{~N}$ is determined. Then,the force is applied on the crystal resonator by gradually rotating the screw. The force will be shown on the digital indicator.

\section{EXAMPLE EXPERIMENTAL RESULTS}

Example experimental results have been obtained for an AT-cutquartz resonator. The diameterand thickness ofthis quartz resonator is $13.97 \mathrm{~mm}$ and $0.274 \mathrm{~mm}$ relatively.Fig.6shows the defined azimuth angles where the tips of the clamps contact and apply force on the resonator. Because of the physical shape of the measured resonator, the azimuth angles for the experiment are defined at these values: $0^{0}, 30^{\circ}, 50^{\circ}, 70^{\circ}, 90^{\circ}, 110^{\circ}, 130^{\circ}, 150^{\circ}$, and $180^{\circ}$. The position $90^{\circ}$, which is at the electric poles' position, can be measured by moving the wires of the holder. The load is increased from $0 \mathrm{~N}$ to $20 \mathrm{~N}$ with $5 \mathrm{~N}$ increments.

Fig. 7 and Fig. 8 show the force-frequency relation in a quartz resonator at angles $0^{0}$ and $110^{\circ}$. In Fig. 7, at azimuth angle $0^{0}$, the resonance frequency increases when the force is increased. Meanwhile, in Fig. 8, at azimuth angle $110^{\circ}$, the resonance frequency decreases when the force is increased.The force sensitivity coefficients $\mathrm{K}_{\mathrm{f}}$ arecalculatedbased on Eq. (2) and plotted in Fig. 9. This result shows a good agreement with peers' measurement results in [9], [10].

\section{CONCLUSION}

An apparatus has been designed and built to precisely measure the force-frequency relation and the force sensitivity coefficient $\mathrm{K}_{\mathrm{f}}$ of crystal resonators. The apparatus includes two key improvements tothe scientific instrumentation: 1) better input force resolution and2) simplicity for force alignment. The apparatus has been used successfully to measure the force- 
frequency relation and force sensitivity coefficient $\mathrm{K}_{\mathrm{f}} \mathrm{of}$ a quartz resonator. Future work would involveimprovingthe crystal resonator'sholder.

\section{ACKNOWLEDGMENTS}

The authors thank Mr. Kurt of the UNT Physics Machine shop for his help in fabricating the apparatus. The research is sponsored by the National Science Foundation under grant number 1335354. 


\section{REFERENCES}

[1] J. A. Kosinski, R. A. Pastore Jr., X. M. Yang, J. S. Yang, and J. A. Turner, "Stress-induced frequency shifts in langasite thickness-mode resonators," inProc. IEEE Int. Frequency Control Symp., pp. 716-722, 2003.

[2] B. K. Sinha, "Stress-induced frequency shifts in thickness-mode resonators," inProc. IEEE Int. Frequency Control Symp., pp. 813-818, 1980.

[3] A. Ballato, E. P. EerNisse, and T. Lukaszek, "The force-frequency effect in doubly rotated quartz resonators,” in Proc. IEEE Int. Frequency Control Symp., pp. 8-16, 1977.

[4] C. Barthod, "New force sensor based on a double ended tuning fork," in Proc. IEEE Int.Frequency Control Symp., pp. 74-78, 2000.

[5] E. P. EerNisse and R. B. Wiggins, "Review of Thickness-Shear Mode Quartz Resonator Sensors for Temperature and Pressure,"IEEE Sensor J., vol. 1, no. 1, pp. 79-87, 2001.

[6] J. M. Ratajski, "Force-frequency coefficient of singly rotated vibrating quartz crystals," IBM J. Res. Dev., vol. 12, no. 1, pp. 92-99, 1968.

[7] E. P. EerNisse, "Temperature dependence of the force frequency effect for the AT-, FC-, SC-, and rotated X-cuts," in Proc. IEEE UltrasonicsSymp., pp. 426-430, 1980.

[8] J. J. Boy, R. J. Besson, E. Bigler, R. Bourquin, and B. Dulmet, “Theoretical and experimental studies of the force-frequency effect in BAW LGS and LGT resonators," inProc. IEEE Int. Frequency Control Symp., pp. 223-226, 2001.

[9] Y. Kim, A. Ballato, "Force-frequency effect of Y-cut langanite and Y-cut langatate," IEEE Trans. Ultrason. Ferroelectr. Freq. Control, vol. 50, no. 12, pp. 1678-1682, 2003. 
[10] J. M. Ratakski, "The force sensitivity of AT-cut quartz crystal," $20^{\text {th }}$ Annual Symp.on Freq. Control, pp. 33-49, 1966.

[11] H.Zhang, J.A. Turner,J.S.Yang, and J.A. Kosinski, "Force-frequency effect of thickness mode langasite resonators," Utrasonics, 50, pp.479-490, 2010.

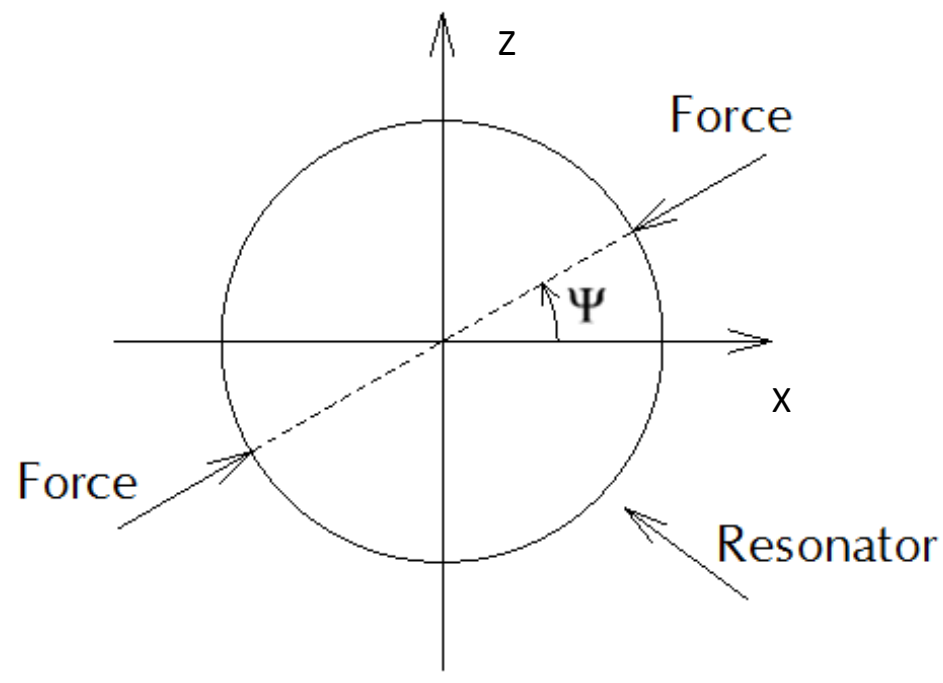

Fig. 1: Illustrationof force application and azimuth angle $\psi$ 


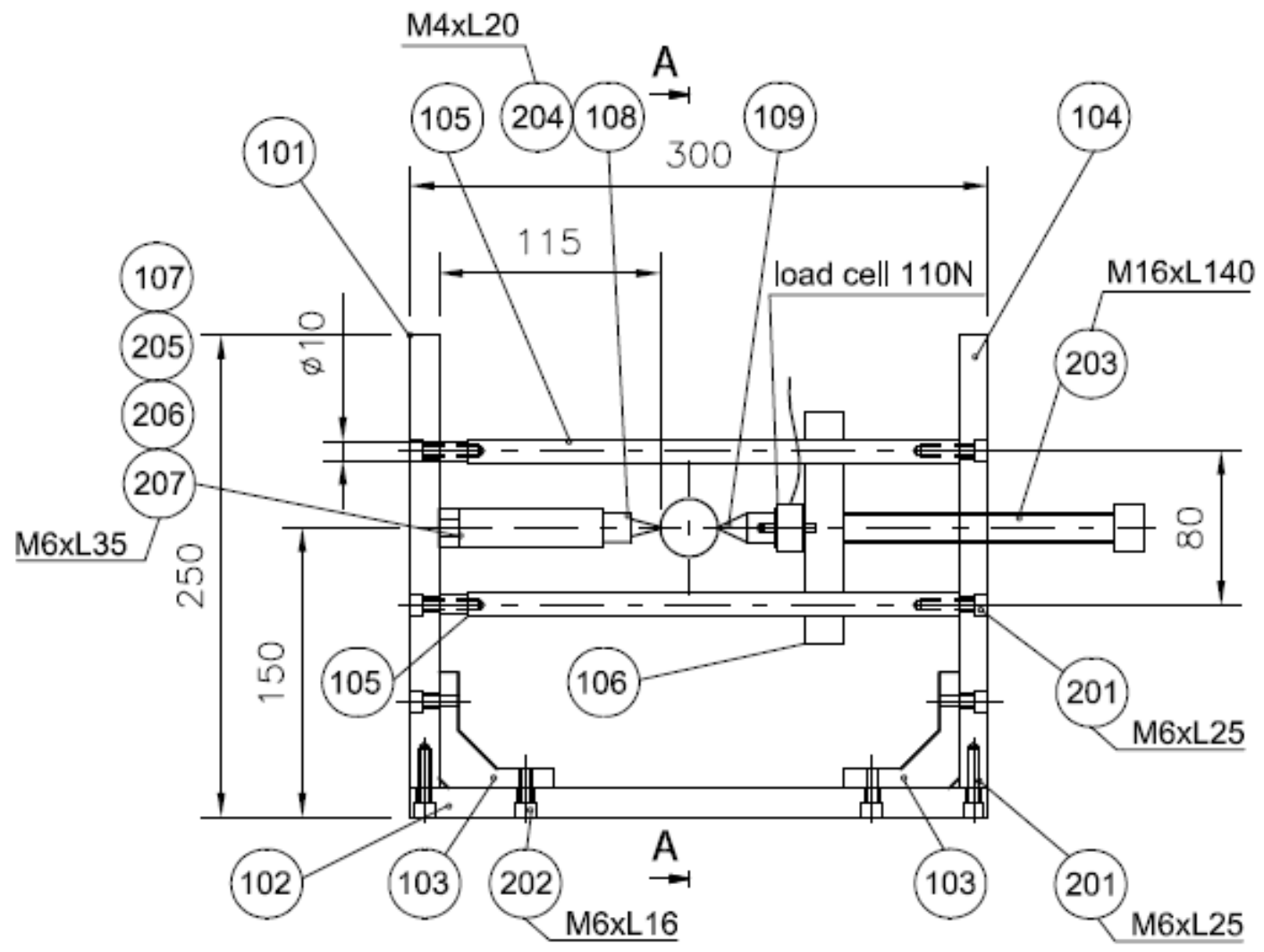

Fig. 2: New loading device assembly 


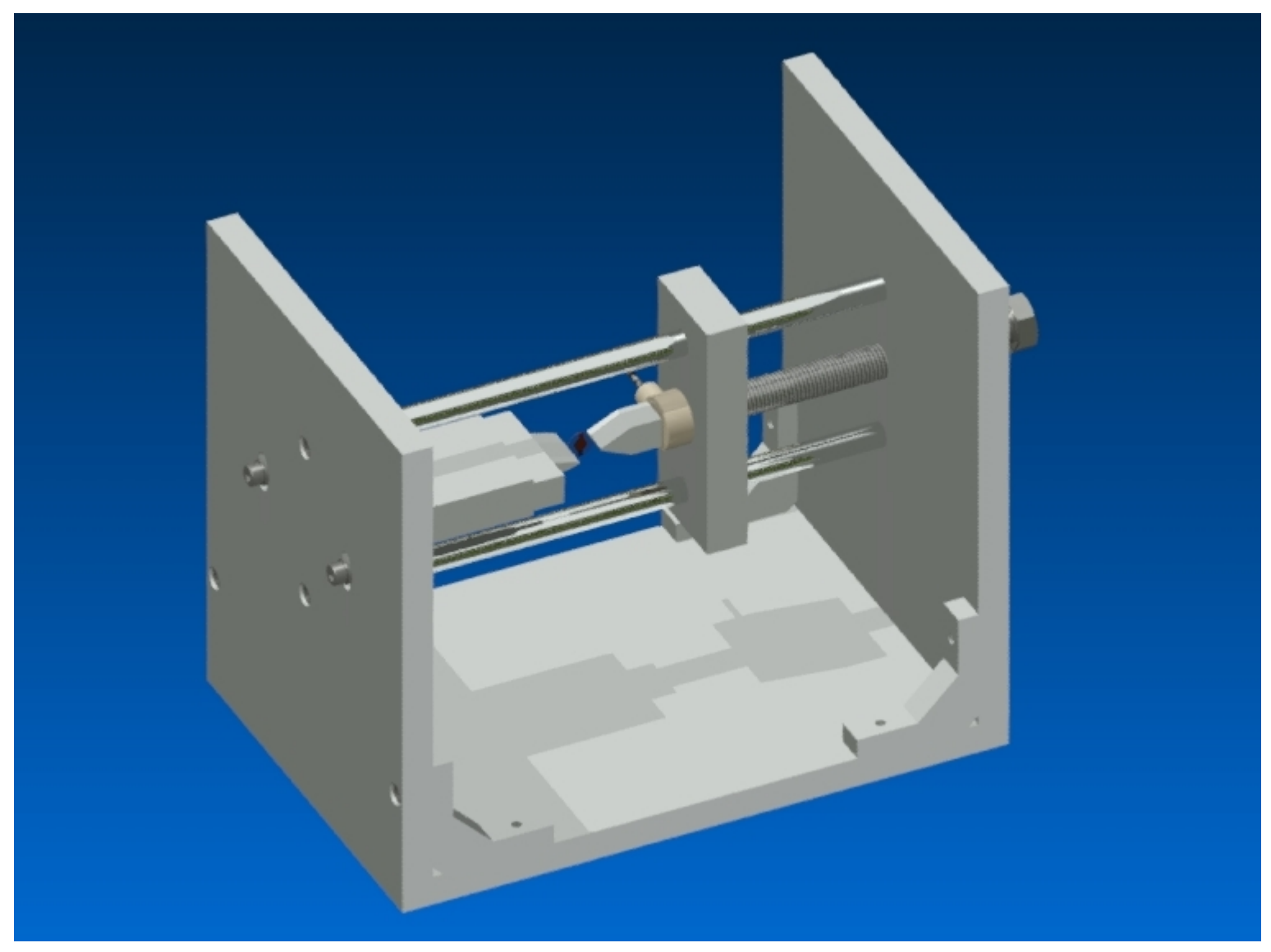

Fig. 3: Isometric view of a 3D model of the new loading device 


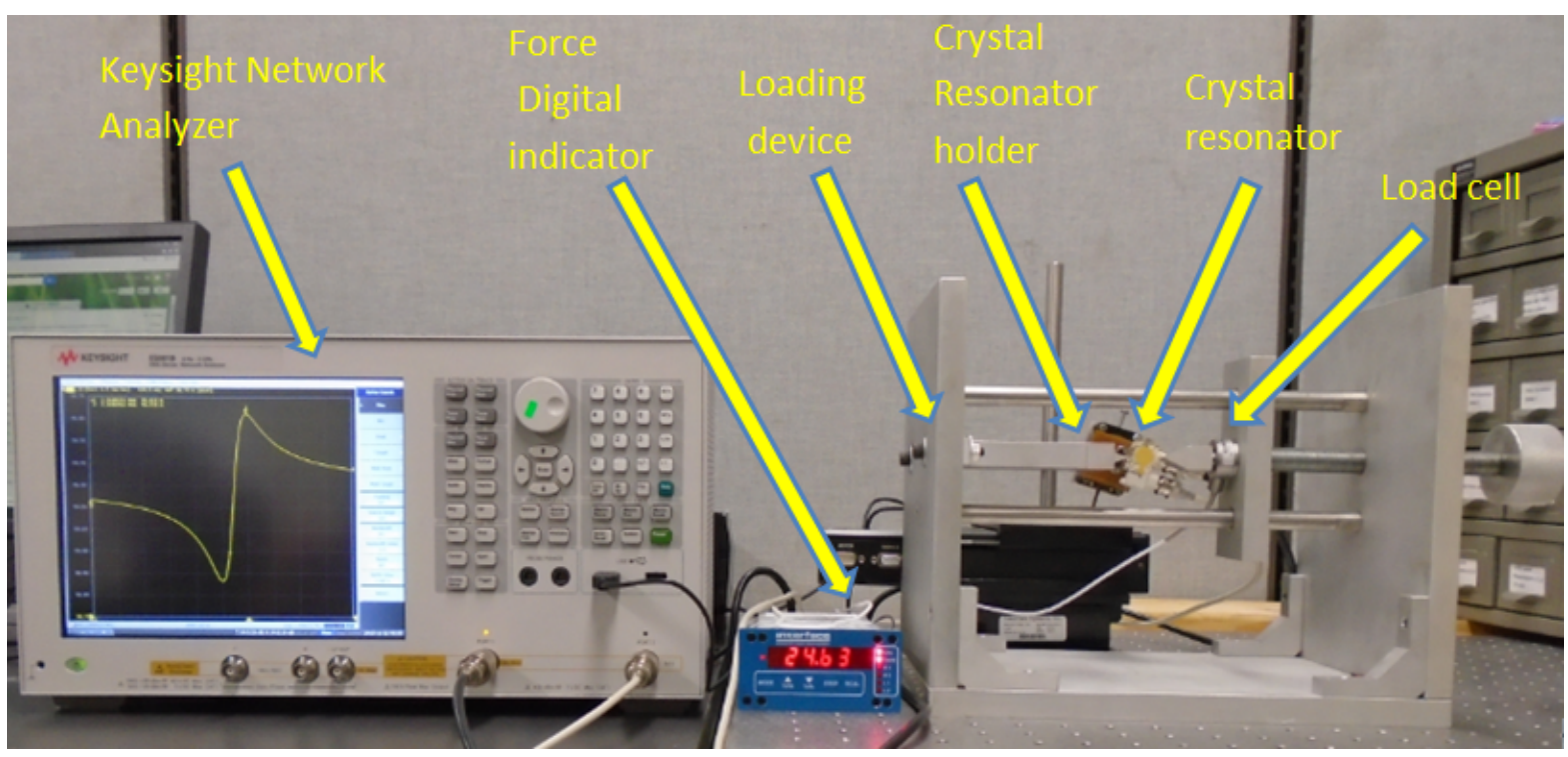

Fig. 4: Description of experiment setup 


\section{Resonance frequency}

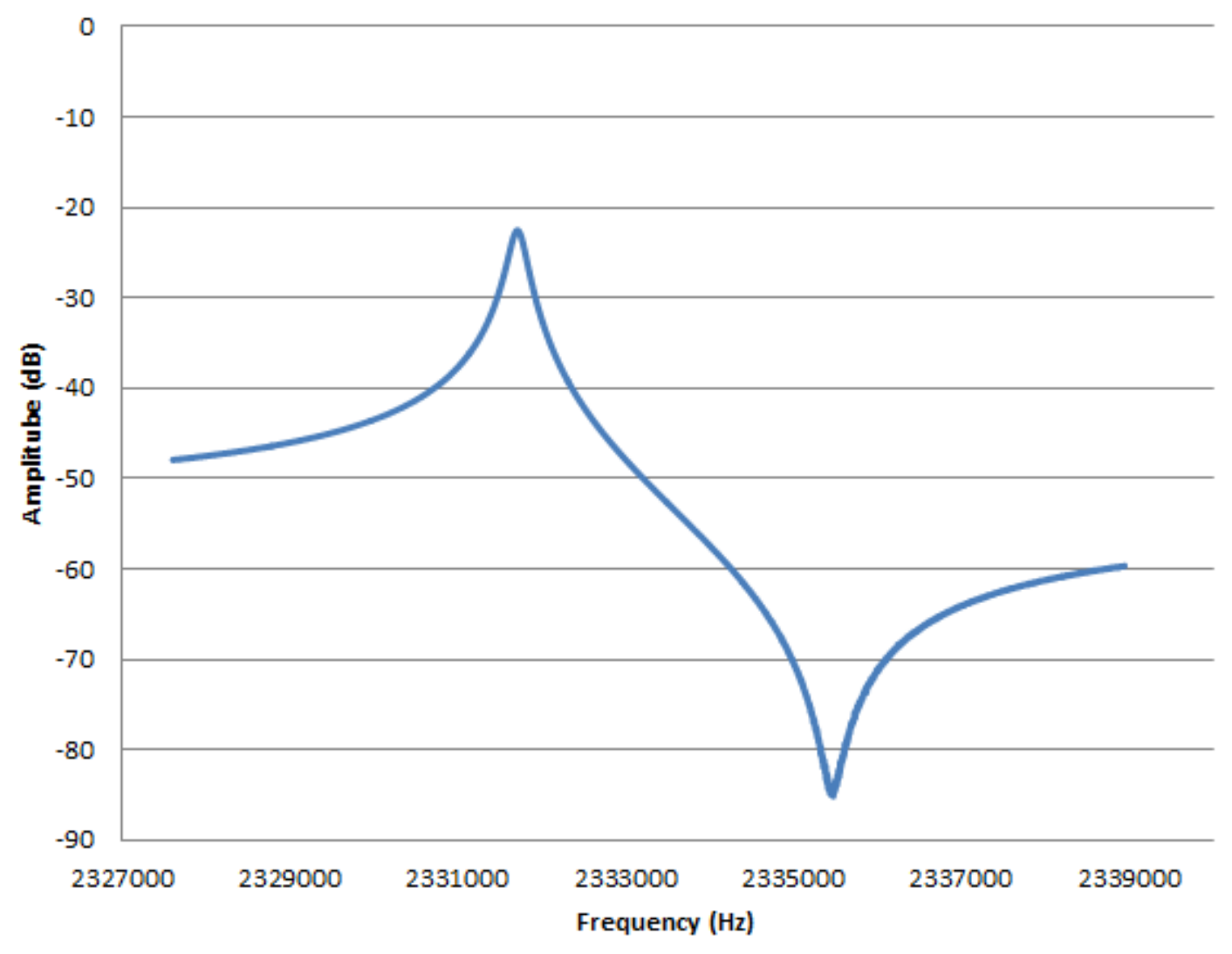

Fig. 5: Typical graph at resonance frequency of a crystal resonator 


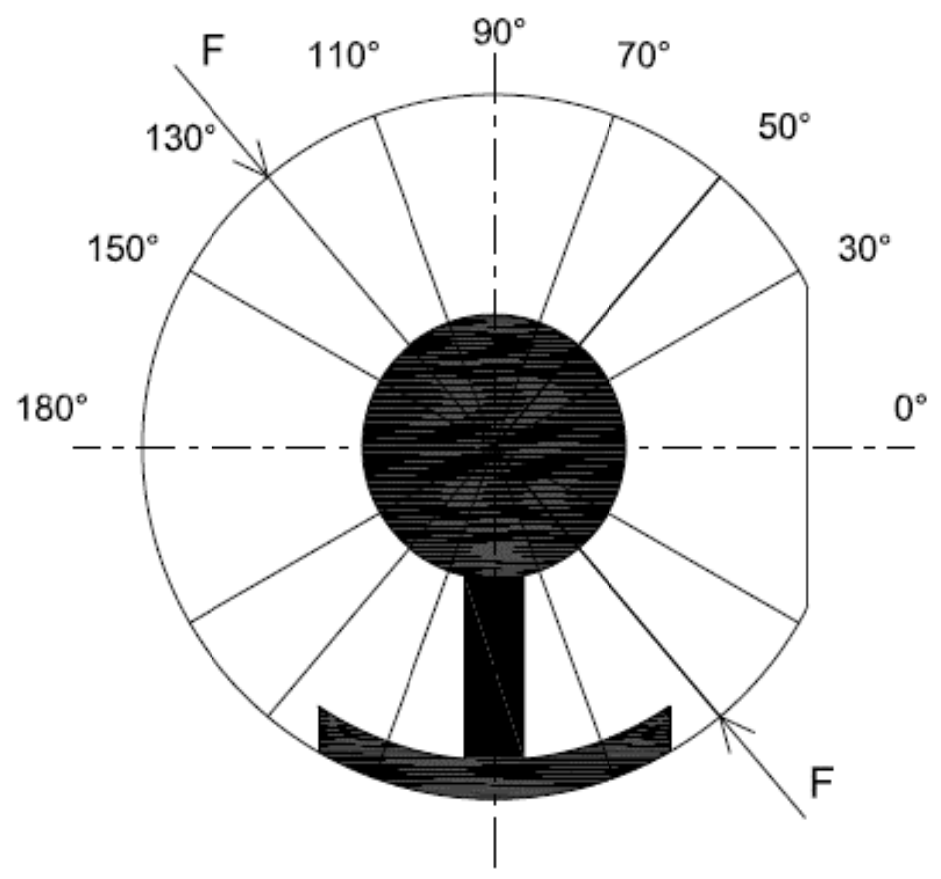

Fig. 6: Definition of azimuth angles for the experiment 


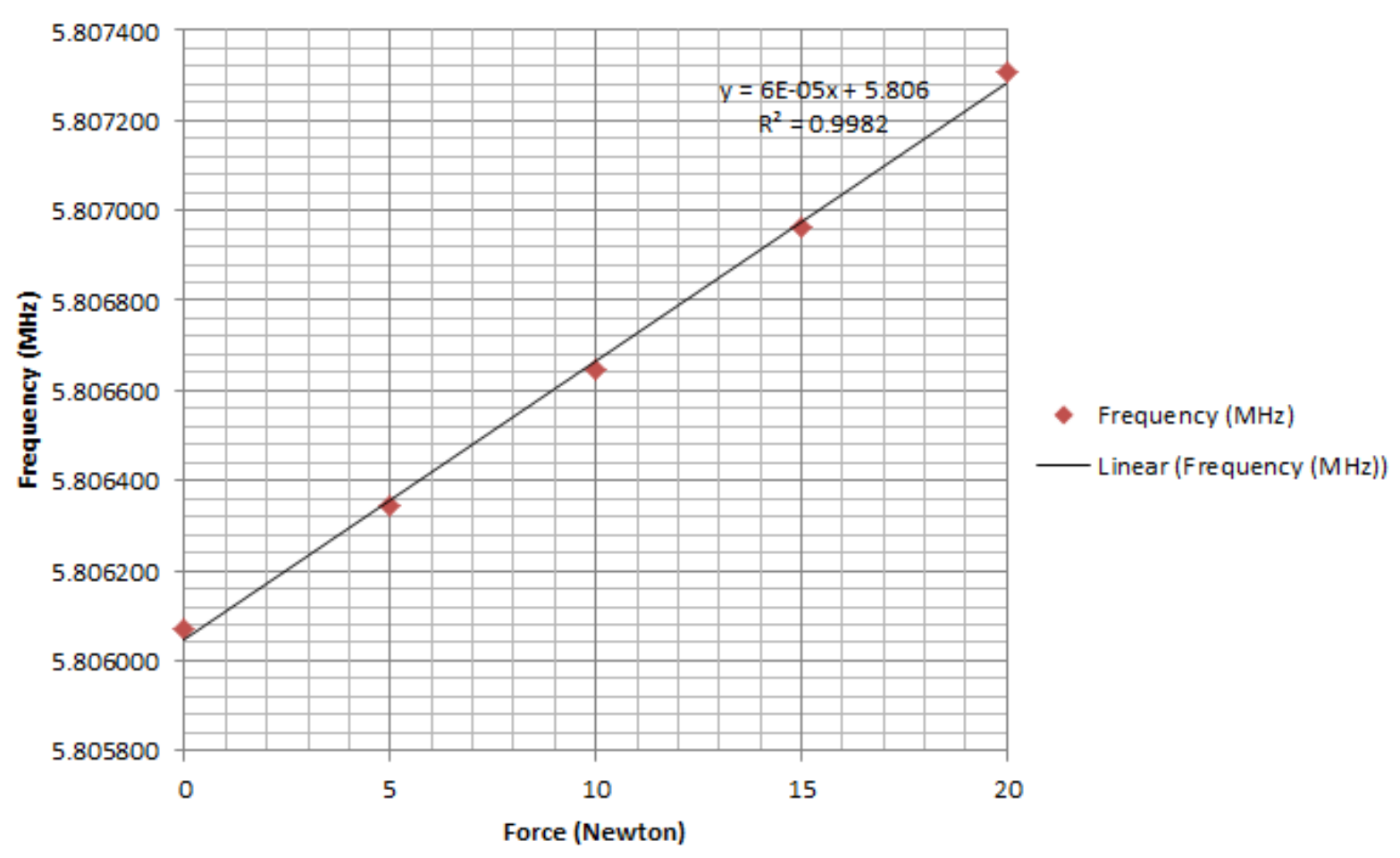

Fig. 7: Typical measurement result for the force vs. frequency relation at azimuth angle $0^{0}$ 


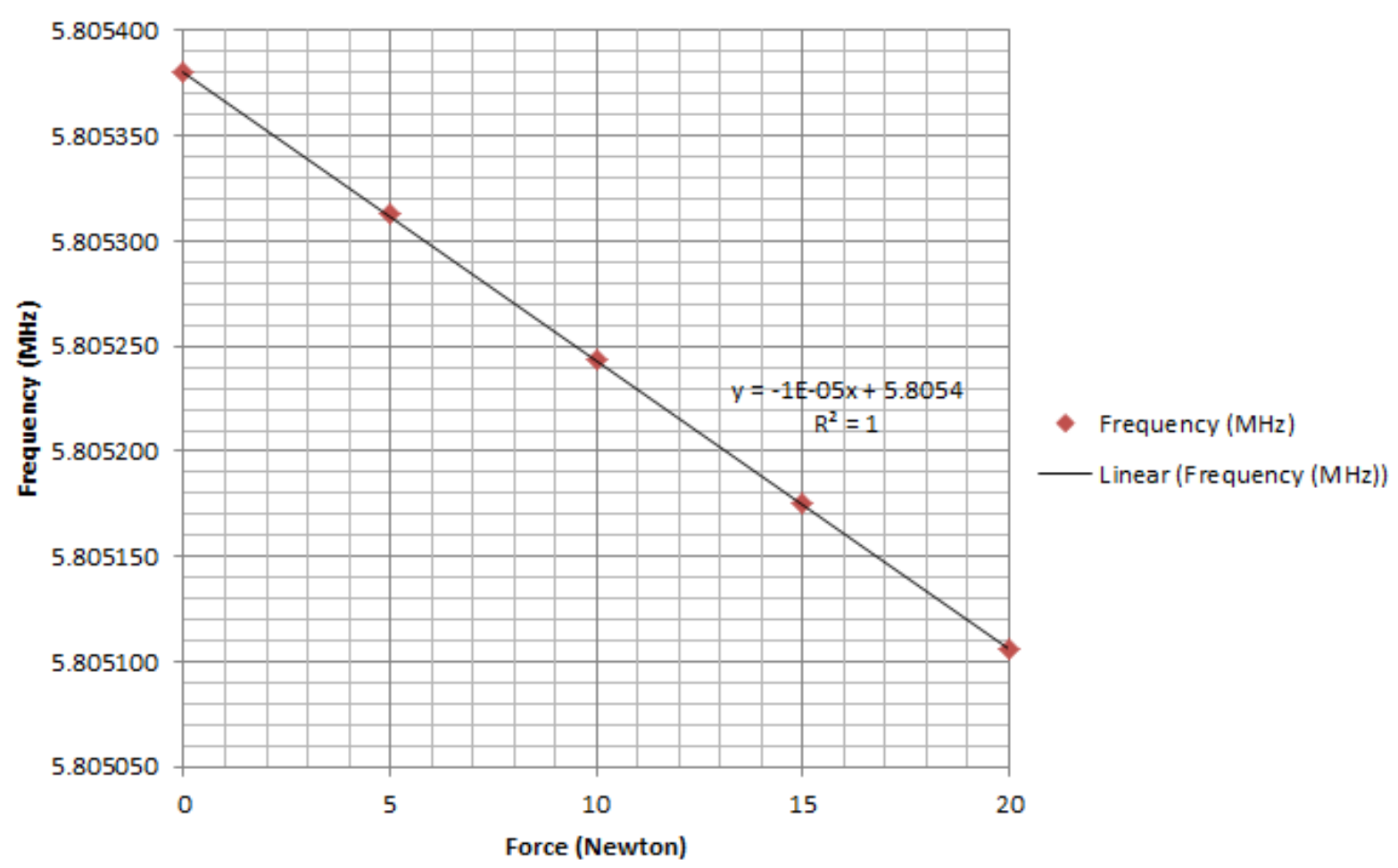

Fig. 8: Typical measurement result for the force vs. frequency relation at azimuth angle $110^{\circ}$ 


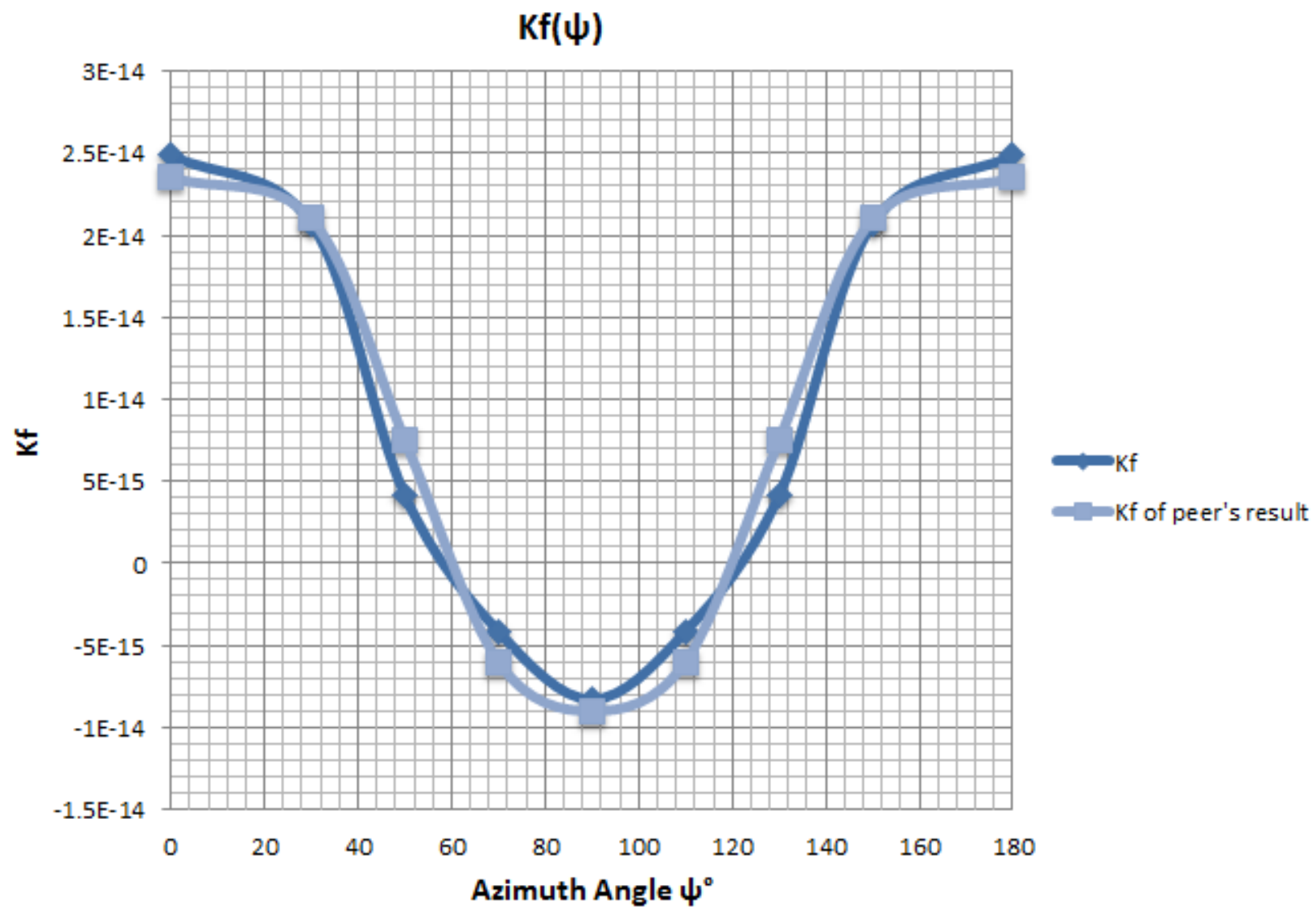

Fig. 9: The curve $K_{f}$ of a quartz resonator from the experiment vs. peer's curve $K_{f}$ 